\title{
Ultrasound v. sham ultrasound for experimentally induced delayed- onset muscle soreness: A double-blind, randomised controlled trial
}

\author{
R Parker, ${ }^{1}$ PhD (Psych); V J Madden, ${ }^{2}$ BSc (Phys) \\ ${ }^{1}$ Department of Health and Rehabilitation Sciences, Faculty of Health Sciences, University of Cape Town, South Africa \\ 2 Sansom Institute for Health Research, University of South Australia, Adelaide, Australia
}

Corresponding author: R Parker (romy.parker@uct.ac.za)

\begin{abstract}
Background. Therapeutic ultrasound (US) is an electrophysical therapy that is commonly used by sports physiotherapists, but its mechanism of action is unclear. There is little evidence that US therapy is more effective than sham US therapy, and any clinical benefits may be due to a placebo effect.

Objective. To investigate whether US has a specific effect that renders it effective in its own right, or whether its effect is placebo driven. Methods. In a double-blind controlled trial, delayed-onset muscle soreness (DOMS) was experimentally induced in both bicep muscles of 15 females. Sham US was applied to one bicep ( $n=15$ biceps) and pulsed active US to the other bicep ( $n=15$ biceps) of each participant, 48 and $72 \mathrm{~h}$ after induction of DOMS. Primary and secondary outcomes were pain reported on the McGill Pain Questionnaire (MPQ) and range of movement (ROM) (elbow extension) measured by goniometry, respectively.

Results. Results showed significant improvements in pain and ROM over the intervention periods, but there was no difference between interventions.

Conclusion. US and sham US therapy improve pain equally when treating DOMS of the biceps in the context of a therapeutic encounter. This analgesic effect is placebo driven. Clinicians can influence the analgesic effect of US by managing the therapeutic context. Management of patients' anxiety may also boost the analgesic effect of US.
\end{abstract}

S Afr J SM 2014;26(4):99-103. DOI:10.7196/SAJSM.535

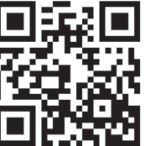

Therapeutic ultrasound (US) is an electrophysical therapy that is commonly used by sports physiotherapists, but its mechanism of action is unclear. ${ }^{[1]}$ There is little evidence that US therapy is more effective than sham US therapy, and any clinical benefits may be due to a placebo effect. ${ }^{[2]}$

The term 'placebo effect' is used when a treatment that is known to have no specific physiological efficacy produces a positive therapeutic outcome. It is heavily reliant on the context of treatment and on the patient's expectation of benefit. ${ }^{[3]}$ Physiotherapists enhance placebo value by using positive therapeutic relationships and educating their patients about the anticipated effects of treatment. ${ }^{[4]}$

Delayed-onset muscle soreness (DOMS) is pain or discomfort that develops in muscles after exercise that is unfamiliar. ${ }^{[5]}$ DOMS is self-limiting and easily induced experimental setting. Clinical findings include muscular tenderness and loss of range of movement (ROM), flexibility and strength. Symptoms develop gradually and peak after $24-48 \mathrm{~h}$, resolving within $72 \mathrm{~h} .^{[6]}$ The efficacy of US in treatment of DOMS has been investigated by a few studies of poor methodological quality. ${ }^{[2]}$

\section{Methods}

This study investigated the effect of US in the treatment of DOMS, and was designed to explore whether US has a specific effect, or whether it works via a placebo effect. A double-blind controlled trial was designed to compare the effects of active US v. sham US on the symptoms of experimentally induced DOMS of the biceps muscle group. The primary outcome was pain, measured by the McGill
Pain Questionnaire (MPQ) ${ }^{[7]}$ the secondary outcome was elbow ROM, measured with a goniometer. ${ }^{[8]}$ The study was conducted in accordance with the Declaration of Helsinki and approved by the institutional research ethics committee.

\section{Participants and setting}

Healthy female physiotherapy students at the University of Cape Town were invited to participate. Exclusion criteria were preexisting medical conditions for which US is contraindicated, preexisting upper limb discomfort or injury, prior experience of US treatment for DOMS or altered test results for skin hot/cold or sharp/ blunt sensations. Twenty undergraduate students, aged 18 - 25 years, volunteered. Participants were given an information sheet listing the symptoms of DOMS and the study procedures, and then given an opportunity to ask further questions before agreeing to participate. Participants were asked not to take pain medication, perform any strenuous exercise or change their diets during the course of the study. At the end of the study, participants were given information sheets detailing interventions that might help to clear remaining symptoms. Interventions and data collection were conducted at the physiotherapy practical teaching venue of the University of Cape Town.

\section{Outcome measures}

The outcome measures were pain and elbow ROM. Pain was assessed using the MPQ, which has shown good validity and reliability in DOMS-related pain ${ }^{[9]}$ after translation and across cultures. ${ }^{[7]}$ Elbow ROM was measured using standardised goniometry, also shown to be valid and reliable by numerous authors. ${ }^{[9]}$ Goniometry was 
performed while participants sat with the upper arm supported. The goniometer's stationary arm was aligned with the longitudinal axis of the humerus and the moving arm with the longitudinal axis of the forearm. One researcher (Assistant A) took all measurements.

\section{Pilot study}

A pilot study was performed to standardise the methods of DOMS induction and data collection. In the pilot study, an I-KON1011 (Chattanooga Ltd, USA) isokinetic machine was used to induce DOMS, but this was found to be ineffective. It was replaced by the following eccentric exercise protocol described by Stay. ${ }^{[10]}$ Participants performed concentric and eccentric bicep curls using a dumbbell. Participants performed four sets of 10 repetitions (or to muscle failure) at $80 \%$ of their one-repetition maximum (1RM). This was followed by four sets of 10 repetitions (or to muscle failure) of eccentric contractions at 100\% of their 1RM. During the eccentric contraction, participants were instructed to slowly lower the dumbbell from full elbow flexion to full extension over $5 \mathrm{~s}$, with Assistant $\mathrm{C}$ returning the weight to the starting position. Participants rested for $1 \mathrm{~min}$ between each set. With the new protocol, pilot participants reported maximal symptoms $48 \mathrm{~h}$ after exercise.

\section{Procedure}

The experimental procedure is illustrated in Fig. 1.

\section{Baseline data collection $\left(T_{0}\right)$}

Baseline measures of pain and elbow ROM were recorded for both biceps (left and right) of each participant before DOMS was induced $\left(\mathrm{T}_{0}\right)$.

\section{Induction of DOMS $\left(T_{0}\right)$}

Before DOMS was induced, a researcher read a prepared script to the participants to remind them of the symptoms of DOMS. Participants were then asked to perform bilateral, resisted concentric and eccentric bicep curls according to the protocol previously described. ${ }^{[10]}$

Following this exercise bout, participants were asked to return for US treatment at 48 and $72 \mathrm{~h}$.

Ultrasound ( $T_{48}$ and $\left.T_{72}\right)$

US Sonoplus 190 machines were calibrated and used with an appropriate coupling gel.
The same two researchers (Assistants B and C) administered all US interventions.

At $48 \mathrm{~h}\left(\mathrm{~T}_{48}\right)$, a script explaining the upcoming and ROM were measured by Assistant A.

Machine A delivered active US at $1 \mathrm{Mhz}$, $0.4 \mathrm{~W} / \mathrm{cm}^{2}$, pulsed 1:4, for $7 \mathrm{~min}$. Machine B delivered a sham dose (intensity set to 0 $\mathrm{W} / \mathrm{cm}^{2}$ ) for the same time period. Assistant D set up the US machines behind a screen and all instrumentation was covered so that the researchers operating the US machines and all participants were blinded as to which machine delivered which dose.

Assignment of machine to biceps was quasi-random. Assistant $\mathrm{E}$ allocated the first participant's right arm to one intervention by coin toss, and subsequent participants' arm allocations were alternated.

Both arms were treated concurrently (one with Machine B and one with Machine A). The same two researchers administered treatment with the same machines at $48\left(\mathrm{~T}_{48}\right)$ and $72 \mathrm{~h}$ $\left(\mathrm{T}_{72}\right)$. This consistency was maintained to standardise treatment and minimise bias.

\section{Data collection}

Pain and ROM were re-assessed before and after each intervention session by a single procedure was read to the participants. Pain

researcher (Assistant A), who was blinded as to which arm received active or sham treatment.

\section{Statistical analysis}

A $30 \%$ reduction in pain was considered clinically significant. ${ }^{[11]} \mathrm{A}$ power calculation with $p<0.05$ suggested a sample of 15 participants ( $N=15$ biceps per group) to detect this change, with a power of 0.86 .

Data were recorded using Microsoft Excel (2003) and analysed using SPSS Statistics version 21 (IBM, USA). Kolmogorov-Smirnov tests found that the data were normally distributed. Repeated measures analyses of variance (ANOVA) with a priori comparisons were used, for both outcomes, to compare each time point with the preceding one. Statistical significance was set at $p<0.05$. Results were presented using means and standard deviations. Mauchly's test was used to assess the assumption of sphericity. Where sphericity was violated, degrees of freedom were corrected using the Greenhouse-Geisser estimates of sphericity and the corrected value was reported.

\section{Results}

\section{Demographics}

Twenty female students volunteered for the study. One was excluded because of recently

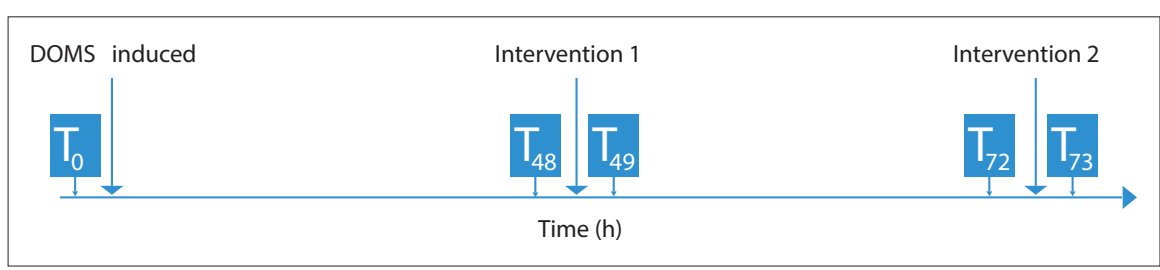

Fig. 1. The experimental procedure. (DOMS = delayed-onset muscle soreness. $)$

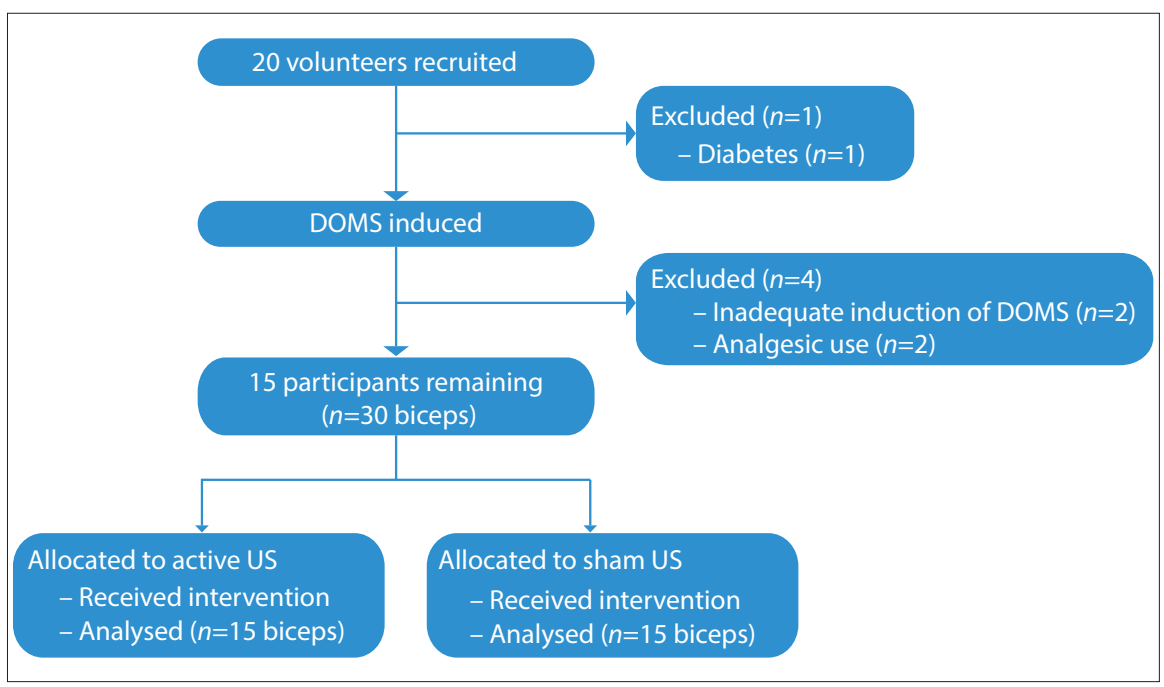

Fig. 2. Breakdown and allocation of research volunteers. (DOMS = delayed-onset muscle soreness; US = ultrasound .) 
diagnosed diabetes. Two participants were excluded because DOMS was not adequately induced (they had no pain or loss of ROM $48 \mathrm{~h}$ after the exercise bout), and a further two for using analgesics.

The final sample comprised 15 participants: 15 biceps in the experimental group and 15 in the control group (Fig. 2).

\section{Effect of US v. sham US on DOMS symptoms}

The changes in pain over time are shown in Fig. 3. Repeated measures 2 (intervention: active or sham US $) \times 4\left(\mathrm{~T}_{48}, \mathrm{~T}_{49}, \mathrm{~T}_{72}, \mathrm{~T}_{73}\right)$ ANOVA showed that the type of intervention had no effect on pain $(p=0.884)$. However, pain differed over time $\left(\mathrm{F}_{(1.68,23.53 .)}=6.94 ; p=0.006\right)$. Contrasts showed that pain decreased significantly from $\mathrm{T}_{48}$ to $\mathrm{T}_{49}\left(\mathrm{~F}_{(1,14)}=7.35\right.$; $p=0.017)$, and from $\mathrm{T}_{72}$ to $\mathrm{T}_{73}\left(\mathrm{~F}_{(1,14)}=12.27\right.$; $p=0.004)$, revealing that the two interventions were equally effective on both treatment occasions. Contrasts also showed that pain did not change between interventions from $\mathrm{T}_{49}$ to $\mathrm{T}_{72}(p=0.59)$. There was no interaction effect between intervention and time.

The changes in ROM over time are shown in Fig. 4. Repeated measures 2 (intervention) $\times 5\left(\mathrm{~T}_{0}, \mathrm{~T}_{48}, \mathrm{~T}_{49}, \mathrm{~T}_{72}, \mathrm{~T}_{73}\right)$ ANOVA showed that the type of intervention had no effect on ROM $(p=0.198)$. However, ROM did change over time $\left(\mathrm{F}_{(2.32,32.52)}=18.91 ; p=0.0001\right)$. Contrasts showed that ROM diminished from baseline to $\mathrm{T}_{48}\left(\mathrm{~F}_{(1,14)}=87.68 ; p=0.0001\right)$, and then increased over the first intervention period from $\mathrm{T}_{48}$ to $\mathrm{T}_{49}\left(\mathrm{~F}_{(1,14)}=11.32 ; p=0.005\right)$. $\mathrm{ROM}$ did not change over the second intervention period ( $p=0.09$ ), or between interventions $(p=0.67)$. There was significant interaction effect $\left(\mathrm{F}_{(4,56)}=2.91 ; p=0.030\right)$. Contrasts for this interaction were marginally significant at one level: $\mathrm{T}_{49} \mathrm{v} \cdot \mathrm{T}_{72}\left(\mathrm{~F}_{(1,14)}=4.66 ; p=0.049\right)$. Over this time period, ROM decreased in the active US group and increased in the sham US group.

\section{Discussion}

\section{Natural history or placebo effect?}

This study used a double-blinded, withinsubject design to investigate the efficacy of sham $v$. active US interventions for decreasing pain related to DOMS of the biceps. Participants received each intervention twice: at $48 \mathrm{~h}$ and $72 \mathrm{~h}$ after induction of DOMS.

Participants reported decreased pain immediately after intervention $\left(\mathrm{T}_{48}-\mathrm{T}_{49}\right.$ and $\mathrm{T}_{72}-\mathrm{T}_{73}$ ), regardless of whether active or sham US was used. In contrast, participants

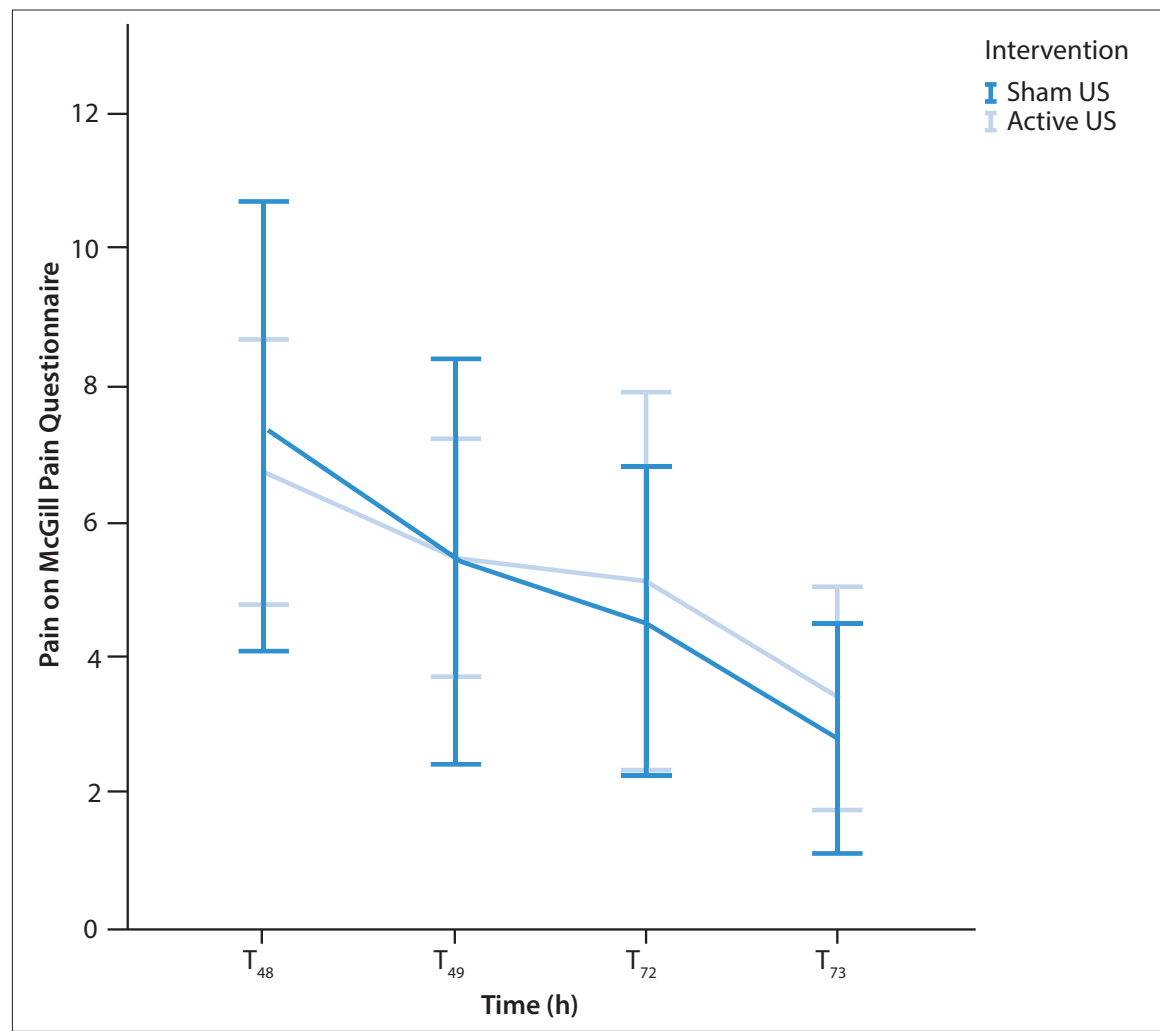

Fig. 3. Changes in pain over time. (US = ultrasound.)

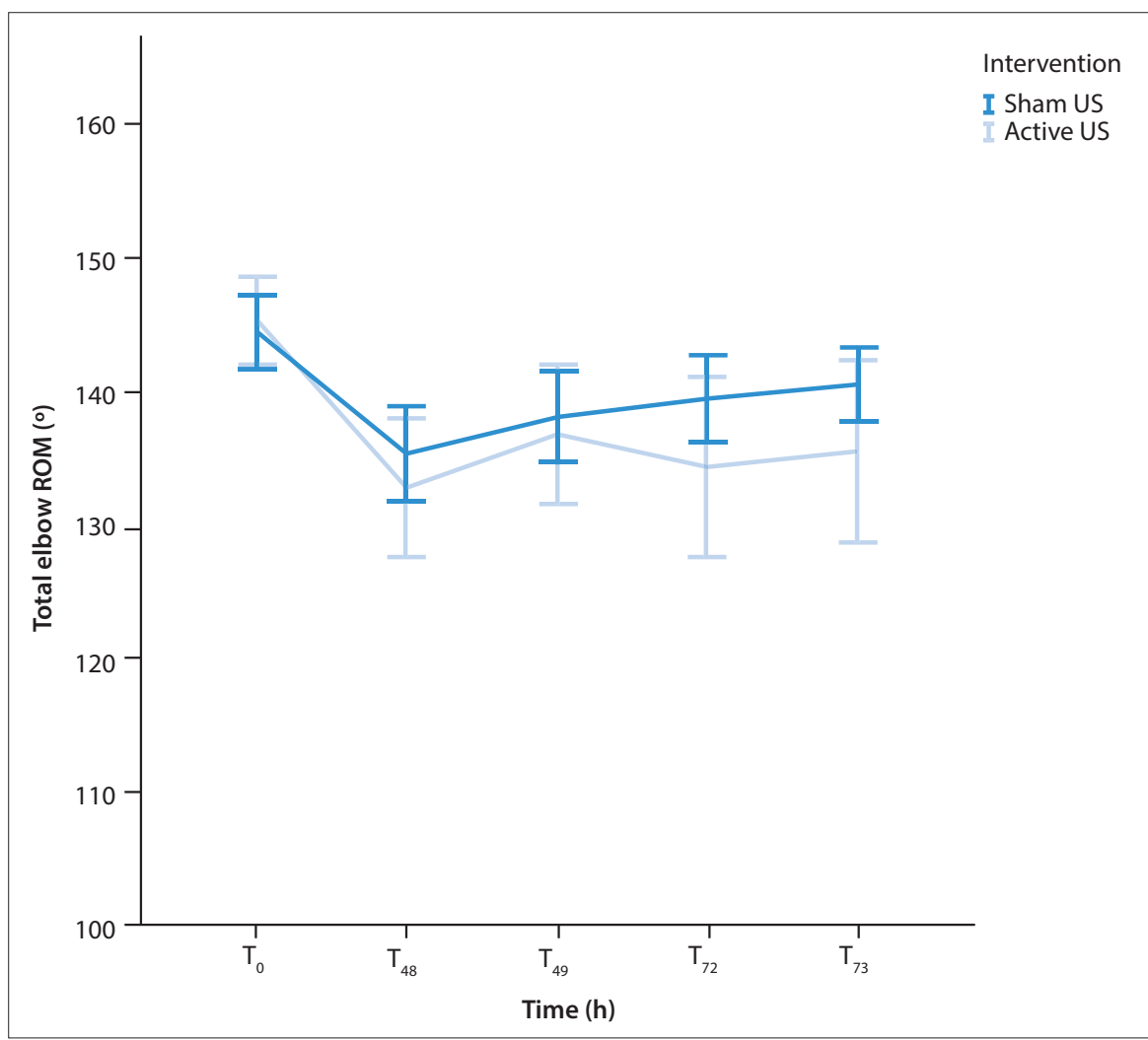

Fig. 4. Changes in elbow ROM over time. $(R O M=$ range of movement; $U S=$ ultrasound. $)$

did not report decreased pain over the $23 \mathrm{~h}$ between interventions $\left(\mathrm{T}_{49}-\mathrm{T}_{72}\right)$, showing that changes in pain over the intervention periods cannot be attributed to spontaneous 
resolution of DOMS. These results indicate that active and sham US treatments were both equally effective in reducing pain.

Changes in ROM were also unaffected by the nature of the intervention. Participants showed a loss of ROM over the first $48 \mathrm{~h}$, to be expected after the induction of DOMS. They showed a recovery of some ROM during the first intervention and no significant changes thereafter, suggesting that the two interventions were equally effective $48 \mathrm{~h}$ after DOMS induction. These results also revealed a statistically significant difference in recovery pattern between sham- and active-treated groups between interventions $\left(\mathrm{T}_{49}-\mathrm{T}_{72}\right)$, but the difference was too small to be clinically relevant.

The comparable efficacy of active US and sham US for decreasing pain and improving ROM when applied to opposite arms of the same participant suggests a mechanism that is systemic rather than local.

\section{Other studies demonstrating a strong placebo effect of US}

Our results corroborate those of Hashish et al. ${ }^{[12]}$ who found sham US to have comparable or better effects than active US for pain and inflammation. In fact, the efficacy of US for musculoskeletal disorders has been reviewed and subjected to meta-analysis. ${ }^{[13]}$ Again, the results indicated that active US is not appreciably superior to sham US in multiple indicators of recovery, including pain and swelling.

However, the findings are not entirely unequivocal: some studies still contradict this idea. Two out of ten papers reviewed by Robertson $e t$ al. ${ }^{[2]}$ showed that active US was more beneficial than sham US for the treatment of soft-tissue injuries; however, only one of these two studies used pain as an outcome.

The available evidence suggests that any positive therapeutic effect of US treatment for pain lies in its placebo value.

\section{How placebos cause analgesia}

The placebo effect is driven by both opioid and non-opioid mechanisms. ${ }^{[14]}$ When a subject anticipates that pain will be relieved, the endogenous opioid system is activated, causing a powerful analgesic effect. This effect can be blocked by naloxone. ${ }^{[3]}$ The placebo effect can also be induced by classic conditioning when pain reduction is not expected: a conditioned response is first set up by repeated administration of an inert substance

Table 1. Pain reported on the MPQ, by intervention

\begin{tabular}{lll}
\hline & Active US arms, mean (SD) & Sham US arms, mean (SD) \\
\hline $\mathrm{T}_{48}$ & $6.73(3.52)$ & $7.40(6.00)$ \\
$\mathrm{T}_{49}$ & $5.47(3.16)$ & $5.40(5.38)$ \\
$\mathrm{T}_{72}$ & $5.13(5.03)$ & $4.53(4.09)$ \\
$\mathrm{T}_{73}$ & $3.40(2.97)$ & $2.80(3.08)$ \\
$\mathrm{MPQ}=$ McGill Pain Questionnaire; US = ultrasound; SD = standard deviation.
\end{tabular}

Table 2. ROM, by intervention

\begin{tabular}{lll}
\hline & Active US arms, mean (SD) & Sham US arms, mean (SD) \\
\hline Baseline & $145.27(5.80)$ & $144.40(6.00)$ \\
$\mathrm{T}_{48}$ & $132.87(9.42)$ & $135.33(6.39)$ \\
$\mathrm{T}_{49}$ & $136.73(9.13)$ & $138.20(5.92)$ \\
$\mathrm{T}_{72}$ & $134.20(12.16)$ & $139.53(5.71)$ \\
$\mathrm{T}_{73}$ & $135.53(12.41)$ & $140.53(5.08)$ \\
ROM = range of movement; US = ultrasound; SD = standard deviation. &
\end{tabular}

together with an exogenous opioid. After this administration, the inert substance alone will induce analgesia. ${ }^{[3]}$ This conditioned response is only partly blocked by naloxone, indicating that it works via a non-opioid mechanism. ${ }^{[3]}$

The opioid-driven component of placebo analgesia acts via descending painmodulating pathways, involving the rostral anterior cingulate cortex, orbitofrontal cortex, peri-aqueductal grey matter, pons and medulla. ${ }^{[15]}$ These pathways use opioids to increase inhibition at the dorsal horn of the spinal cord. This reduces the amount of nociceptive signal reaching the brain. Endogenous opioids also seem to reduce inflammatory pain in peripheral tissues. Cholecystokinin (CCK) antagonises this entire opioid-mediated process, which corresponds with CCK's known role in states of anxiety: CCK may increase pain when subjects anticipate negative outcomes. ${ }^{[3]}$

The anticipation that underpins opioiddriven placebo analgesia is thought to depend heavily on psychosocial context. The presence of a therapist, the ritual of giving a treatment and the visible presence of therapeutic equipment may influence it. ${ }^{[3]}$ US fits this model: it is a 'hands-on' therapy that requires a therapist to be actively involved throughout the treatment.

In this study, US was delivered for $7 \mathrm{~min}$ with the US transducer head visible to the participants. The information sheet was read to participants before the intervention to reduce expectations regarding the efficacy of US therapy ('Ultrasound is an electrotherapy modality that is commonly used by physiotherapists during treatment sessions. However, there is limited evidence to support its effectiveness in the treatment of DOMS. In this study, the effectiveness of a non-thermal dose of ultrasound on DOMS will be investigated with the aim of increasing the evidence base for the therapeutic use of ultrasound'), but in other respects the psychosocial context was typical of a therapeutic situation. The presence of a therapist and provision of apparent therapy may be enough to overrule a neutral information sheet, thus creating an overall expectation of improvement. Further expectation may have been generated by the fact that the participants in the study were physiotherapy students who had been trained to use US. Although their education and training included critical, evidence-based material on the efficacy of US, simply including the modality in the curriculum may have engendered a belief that it works. Anticipation of a positive outcome would cause an opioiddriven placebo response and reduce pain. ${ }^{[16]}$

\section{Implications for practice}

The ethical considerations arising from studies such as this one have caused some controversy. Is it acceptable to prescribe placebo treatments to patients? The benefits of placebo-induced analgesia have been well documented, ${ }^{[14]}$ so the conflict revolves 
around issues of truthfulness and a patient's autonomy rather than the efficacy of the intervention. Deception may undermine the trust that is so important in a good patient-therapist relationship, and compromise the principles of informed consent. However, deception may not be obligatory, because the therapeutic ritual itself is thought to cause the placebo effect. ${ }^{[14]}$ The power of the therapeutic context has repeatedly been demonstrated in comparisons between hidden and open administration of analgesics: analgesia that is administered openly has a far more powerful effect than the same medication given covertly. ${ }^{[3]}$ What has not yet been well investigated is whether the therapeutic context alone can produce adequate analgesia without a specific therapy. However, medical professionals are well placed to use listening, empathy and patient-centred communication so as to encourage patients to expect positive outcomes. Miller and Colloca ${ }^{[14]}$ argue that it is not necessary to use deception when explaining treatments that are known to be strongly placebo driven. They suggest that a therapist should explain to the patient that studies have found both the active and sham modalities to produce notable and equal improvements. The point is that it doesn't matter which version of intervention the patient receives, as long as they receive one. To be acceptable, this explanation needs to be based on good evidence that sham and active treatments are equally helpful.

\section{Strengths and limitations}

In this study, each participant acted as her own control. This was a notable advantage of the design. This, together with the double blinding, ensured that the centrally controlled, systemic placebo response would be induced equally in both the active US and sham US arms, while local treatment (active US effects) would occur in the active US arm only. The symptoms of DOMS are known to differ between subjects ${ }^{[6]}$ and the subjective experience of pain cannot be compared accurately between different people. Differences in symptoms should therefore be compared in the same person, as was done here.

In this study, the timing of administration of US was based on the findings of the pilot study, not tailored to each participant. Changes in symptoms were not monitored. However, participants in this study acted as their own controls. Since it is unlikely that DOMS would occur asymmetrically in a single participant, any difference in timing should not have influenced the comparisons across time or groups.

Participants' other activities during the study were not controlled or monitored, although participants were asked not to do any strenuous physical activity during the experiment. Varying activity levels could have had an effect on the level of DOMS symptoms, potentially confounding results. Again, marked differences in biceps activity is unlikely to have occurred asymmetrically within a single participant.

Whether the improvement in pain is due to the natural resolution of symptoms or the placebo effect cannot be ascertained without a non-treatment control group. However, the lack of measurable improvement during the 23 -h period between treatment sessions, compared with the significant decrease in pain after each 1-h treatment session, suggests that little of the improvement recorded after treatment was due to spontaneous resolution.

\section{Conclusion}

This study showed that active US ( $1 \mathrm{Mhz}, 0.4 \mathrm{~W} / \mathrm{cm}^{2}$, pulsed 1:4) and sham US (1 Mhz, $0 \mathrm{~W} / \mathrm{cm}^{2}$, pulsed 1:4) therapy produce equivalent improvements in pain when used to treat DOMS of the biceps in the context of a therapeutic encounter. These results suggest that US does reduce pain, but does so via a placebo effect rather than by a local, tissue-specific effect. Clinicians should be guided by this knowledge when choosing between interventions to reduce patients' pain.

Further research is required to determine whether or not the therapeutic context is sufficient to elicit a placebo effect, even in the absence of a specific therapy. However, what is increasingly clear is that nearly every specific therapy also has some placebo value. Clinicians can capitalise on this placebo value with communication strategies that improve the patient's experience of the therapeutic encounter and encourage the patient to expect a positive outcome. Because anxiety interferes with the opioid-mediated mechanism of placebo analgesia, we suggest that clinicians will also need to recognise and address patients' anxiety if the placebo value is to be harnessed. Capitalising on the placebo value of the therapeutic encounter will boost the analgesic efficacy of therapeutic interventions and achieve greater benefit for patients.

Acknowledgements. Experiment funding was obtained through the University of Cape Town's undergraduate physiotherapy programme. The authors thank S Bacon, D Gabriel, C le Grange, W Morris, K Wood and $\mathrm{C}$ Ziervogel for their assistance in conducting the study.

\section{References}

1. Baker KG, Robertson VG, Duck FA. A review of therapeutic ultrasound: Biophysical effects. Phys Ther 2001;81(7):1351-1358.

2. Robertson VJ, Baker KG. A review of therapeutic ultrasound: Effectiveness studies. Phys Ther 2001;81(7):1339-1350.

3. Benedetti F. Placebo and endogenous mechanisms of analgesia. Handb Exp Pharmacol 2007;177:393-413. [http://dx.doi.org/10.1007/978-3-540-33823-9_14]

4. Crow R, Gage H, Hampson S, Hart J, Kimber A, Thomas H. The role of expectancies in the placebo effect and their use in the delivery of health care: A systematic review. Health Technol Assess 1999;3(3):1-96.

5. Cheung K, Hume PA, Maxwell L. Delayed onset muscle soreness: Treatment strategies and performance factors. Sports Med 2003;33(2):145-164.

6. Connolly DAJ. Treatment and prevention of delayed onset muscle soreness. J Strength Cond Res 2003;17(1):197-208.

7. Cleather DJ, Guthrie SR. Quantifying delayed-onset muscle soreness: A comparison of unidimensional and multidimensional instrumentation. J Sports Sci 2007;25(8):845 850. [http://dx.doi.org/10.1080/02640410600908050]

8. Rothstein JM, Miller PJ, Roettger RF. Goniometric reliability in a clinical setting. Elbow and knee measurements. Phys Ther 1983;63(10):1611-1615.

9. Craig JA, Bradley J, Walsh DM, Baxter BD, Allen JM. Delayed onset muscle soreness: Lack of effect of therapeutic ultrasound in humans. Arch Phys Med Rehabil 1999;80(3):318-323. [http://dx.doi.org/10.1016/S0003-9993(99)90144-2]

10. Stay JC. Pulsed ultrasound fails to diminish delayed-onset muscle soreness symptoms. J Athl Train 1998;33(4):341-346.

11. Wittink HM, Strassels SA, Carr DB. Health outcomes and treatment effectiveness in pain medicine. In: Wittink HM, Carr DB, eds. Pain Management: Evidence, Outcomes and Quality of Life. Edinburgh: Elsevier, 2008:1-20.

12. Hashish I, Hai HK, Harvey W, Feinmann C, Harris M. Reduction of postoperative pain and swelling by ultrasound treatment: A placebo effect. Pain 1988;33(3):303-311

13. Gam AN, Johannsen F. Ultrasound therapy in musculoskeletal disorders: A metaanalysis. Pain 1995;63(1):85-91.

14. Miller FG, Colloca L. The legitimacy of placebo treatments in clinical practice: Evidence and ethics. Am J Bioeth 2009;9(12):39-47. [http://dx.doi. org/10.1080/15265160903316263]

15. Petrovic P, Kalso E, Petersson KM, Ingvar, M. Placebo and opioid analgesia: Imaging a shared neuronal network. Science 2002;295(5560):1737-1740. [http://dx.doi.org/10.1126/ science.1067176]

16. Price DD, Finniss DG, Benedetti F. A comprehensive review of the placebo effect: Recent advances and current thought. Annu Rev Psychol 2008;59:565-590. [http:// dx.doi.org/10.1146/annurev.psych.59.113006.095941] 